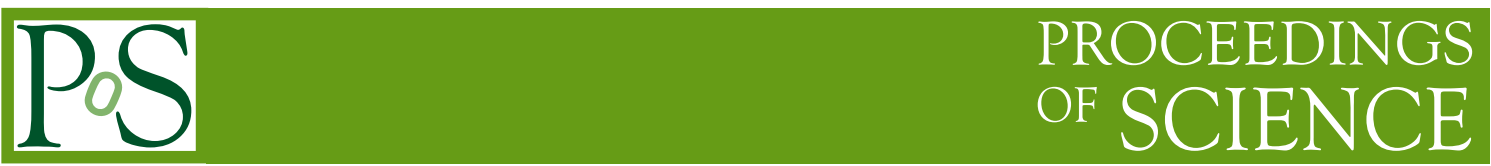

\title{
Performance of the particle flow algorithm in CMS
}

\author{
Florian Beaudette on behalf of the CMS collaboration* \\ CERN,LLR École Polytech., IN2P3-CNRS \\ E-mail: Florian.Beaudettedcern.ch
}

\begin{abstract}
The aim of the CMS particle flow event-reconstruction algorithm is to identify and reconstruct individually each particle arising from the LHC proton-proton collision, by combining the information from all subdetectors. The resulting global event description leads to an improved performance for the reconstruction of jets and MET, and for the identification of electrons, muons, and taus. The $7 \mathrm{TeV}$ jet data, as well as leptons from $\mathrm{J} / \mathrm{Psi}$ and $\mathrm{W}$ are used to finalize the commissioning of the particle-flow algorithm. The efficient reconstruction and identification of photons, charged and neutral hadrons, muons and electrons made possible by the versatility of the CMS apparatus, are shown to perform as expected up to a high level of precision. Results on particlebased jets and missing transverse energy, as well as on muons and electrons obtained through the reconstruction of standard candles are shown.
\end{abstract}

35th International Conference of High Energy Physics - ICHEP2010,

July 22-28, 2010

Paris France

${ }^{*}$ Speaker. 


\section{The Particle Flow algorithm}

In the CMS [1] particle flow algorithm [2], the information coming from the different subdetectors are combined so as to reconstruct individually all the particles resulting from the pp collisions. The resulting list of particles, namely charged hadrons, photons, neutral hadrons, electrons and muons, is then used to reconstruct the jets, the missing transverse energy $\left(E_{\mathrm{T}}^{\mathrm{miss}}\right)$, to reconstruct and identify the taus from their decays products and to measure the isolation of the particles. The algorithm relies on a efficient and pure track reconstruction, on a clustering algorithm able to disentangle overlapping showers, and on an efficient link procedure to connect these building bricks together.

\section{Performance in the simulation}

Typically, the fractions of the jet transverse momentum $\left(p_{\mathrm{T}}\right)$ carried by the charged hadrons, photons, and neutral hadrons are $65 \%, 25 \%$ and $10 \%$ respectively [3]. Therefore, on average, in a jet, $90 \%$ of the energy is determined with a high precision by the tracker or the electromagnetic calorimeter while only $10 \%$ of the energy is affected by the low resolution of the hadron calorimeter. As a result, the jets made of reconstructed particles are expected to be much closer, in energy and direction, to jets made of Monte-Carlo-generated particles than jets made from the sole calorimeter information. The jets in a sample of multijet events are reconstructed with the iterative-cone algorithm which a cone size of 0.5 in the $(\eta, \phi)$ plane, from several types of inputs: all generated stable particles ("genjets"), particles reconstructed by the particle-flow algorithm ("particle-flow jets") and calorimeter towers ("calo-jets"). The jet response, defined as the Gaussian mean of the $\left(p_{\mathrm{T}}^{\text {rec }}-p_{\mathrm{T}}^{\text {gen }}\right) / p_{\mathrm{T}}^{\text {gen }}$ distribution (where "rec" and "gen" hold for reconstructed and generated jets, respectively), is shown in Fig. 1 1 for several $p_{\mathrm{T}}$ bins.

The particle-flow-jet response benefits from the reconstruction of all particles in the event from a combination of all CMS sub-detectors, which ensures that little energy is lost over the whole acceptance, thus giving a good response as shown in Fig. 1 1 a. The particle-flow-jet energy resolutions, obtained by dividing the Gaussian $\sigma$ by the average jet response, in each $p_{\mathrm{T}}$ bin, are compared to the fully corrected calo-jets in Fig. 1 $\mathrm{b}$. Up to three times better resolution for jets is obtained using the particle-flow event reconstruction.

\section{Commissioning with data}

\subsection{Photon and hadron reconstruction}

The building bricks of the algorithm, e.g. the clusters, the tracks, the muons, as well as the linking procedure between these elements have been commissioned. The $\pi^{0} \rightarrow \gamma \gamma$ decays have been used to study the performance of the clusters reconstructed in the electromagnetic calorimeter (ECAL). The photon-pair invariant-mass spectrum resulting in a peak whose fitted mean value agrees with the PDG value within $1 \%$, as well as a remarkable agreement with the simulation, validate the energy corrections applied at low energy to the clusters. The calibration of the hadron calorimeter (HCAL), which is important for the energy determination of the neutral hadrons, has been checked with charged hadrons by comparing the calibrated calorimeter energy to the track 


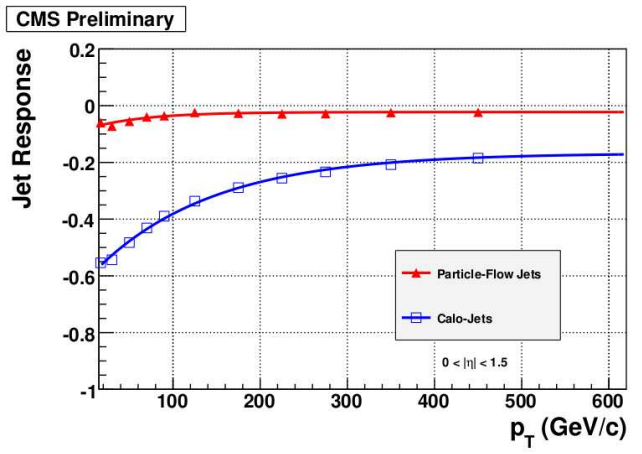

(a)

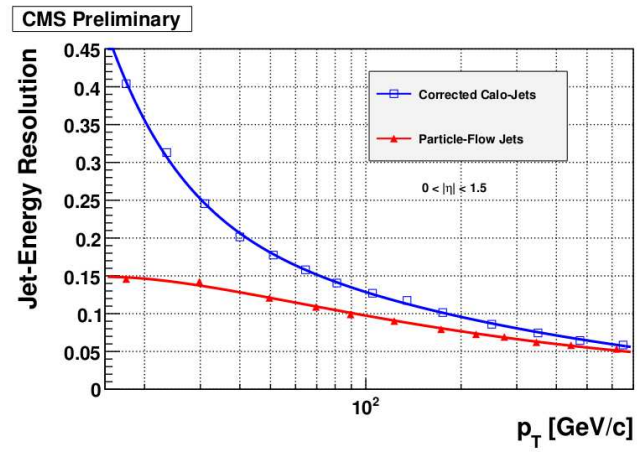

(b)

Figure 1: Jet response (a) and jet-energy resolution (b) as a function of $p_{T}$ in the CMS barrel region $(|\eta|<$ 1.5) for the particle-flow jets (triangles) and the calo-jets (squares).

momentum. Altogether, a satisfying linear behaviour is obtained, with some deviations below the $5 \%$ level, due to the imperfect HCAL calibration.

As a result, taking into account the fractions of the jet energy carried by the different types of particles, together with the current precision of the ECAL and HCAL calibrations, as well as the high precision of the tracker measurement, the legitimate goal for the jet energy scale uncertainty is $1 \%$.

\subsection{Jets and $E_{\mathrm{T}}^{\text {miss }}$}

Events with at least two back-to-back jets with a transverse energy larger than $25 \mathrm{GeV} / \mathrm{c}$ have been selected to commission the performance of the algorithm for the jets and $E_{\mathrm{T}}^{\text {miss }}$ reconstruction. The $p_{\mathrm{T}}$ spectrum of the jets in these events shows a remarkable agreement over more than three orders of magnitude between the data and the simulation. Besides, the invariant mass of the jets, resulting from the combination of their constituents exhibits a similar degree of agreement.

The reconstruction is further commissioned, first by studying the $E_{\mathrm{T}}^{\text {miss }}$ distribution, a quantity which is known to be sensitive to detector effects. In the case of the particle-flow event reconstruction, the $E_{\mathrm{T}}^{\text {miss }}$ is simply the modulus of the vector sum of the reconstructed particle transverse momenta. As visible in Fig. Za, the distribution obtained in the data is reproduced by the simulation with an impressive agreement over several order of magnitudes [3]. Even more challenging, the scalar sum of the particle momenta, $\sum E_{\mathrm{T}}$, for which no noise cancellation can occur, is also faithfully reproduced by the simulation, proving the refined knowledge of the detector included in the simulation.

The superiority of the particle-based determination of the $E_{\mathrm{T}}^{\text {miss }}$ can be established in the data by measuring the width of the distribution of the $E_{\mathrm{T}}^{\text {miss }}$-component projected on one axis as a function of the reconstructed $\sum E_{\mathrm{T}}$. The stochastic term driving the evolution with $\sum E_{\mathrm{T}}$ of the particle-based $E_{\mathrm{X}, \mathrm{Y}}^{\text {miss }}$ resolution is between $50 \%$ and $60 \%$, and is about twice as small as for the calorimeter-based reconstruction, as visible in Fig. Z b b.

\section{Leptons in the particle-flow event reconstruction}

The muons and the electrons are, of course, included in the particle-flow event description. 


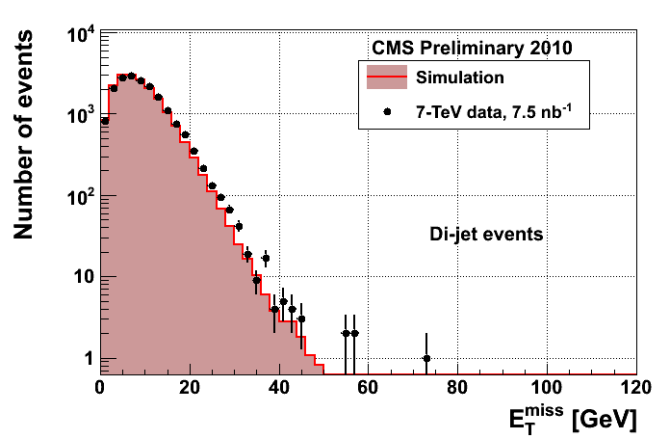

(a)

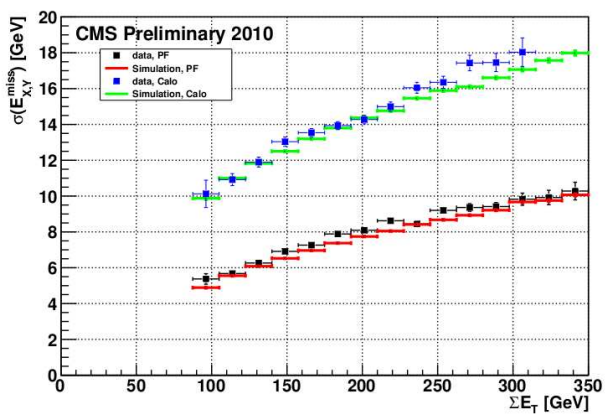

(b)

Figure 2: In di-jet events, distribution of $E_{\mathrm{T}}^{\text {miss }}$ (a) and resolution of $E_{\mathrm{X}, \mathrm{Y}}^{\text {miss }}$ for particle-based (solid symbols) and calorimeter-based (hollow symbols) reconstruction as a function of $\sum E_{\mathrm{T}}$ in di-jet events in the data (b).

The reconstruction of the electrons is a challenge. Indeed the large amount of tracker material (up to two radiation lengths) induces a significant Bremsstrahlung photon emission, which, because of the track curvature in the $3.8 \mathrm{~T}$ magnetic field, can result in a calorimeter energy deposit widely spread in the azimuthal direction. A dedicated algorithm able to reconstruct low momentum and non-isolated electrons has been developed in the context of the particle-flow project [2, 田. It relies primarily on the reconstructed electron track, and identifies the Bremsstrahlung photon clusters in the ECAL by emulating a photon emission for each tracker layer crossed by the track. The identification of the electron is then carried out using a multivariate estimator, $\xi$. A sample of $\mathrm{J} / \Psi \rightarrow \mathrm{e}^{+} \mathrm{e}^{-}$has first been obtained in CMS by selecting events with two particle-flow electrons. The fitted $\mathrm{J} / \Psi$ mass presented in Fig. Ba, agreeing within $1 \%$ with the PDG value, validates the track-calorimeter energy combination used to compute the momentum. This sample has then been used to study the $\xi$-variable unfolding the signal and the background using the sPlot technique [5]. As visible in Fig. 3 $\mathrm{b}$, a satisfactory agreement with the simulation is obtained.

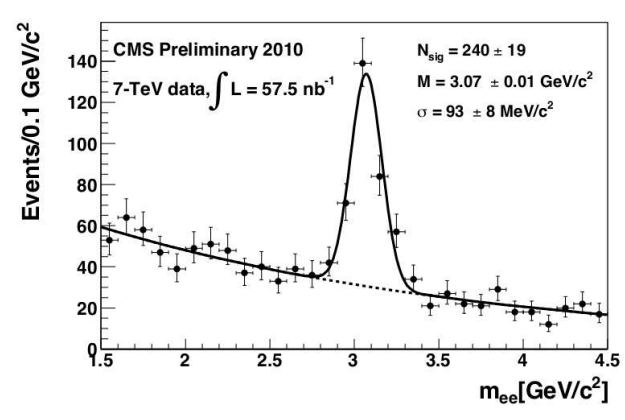

(a)

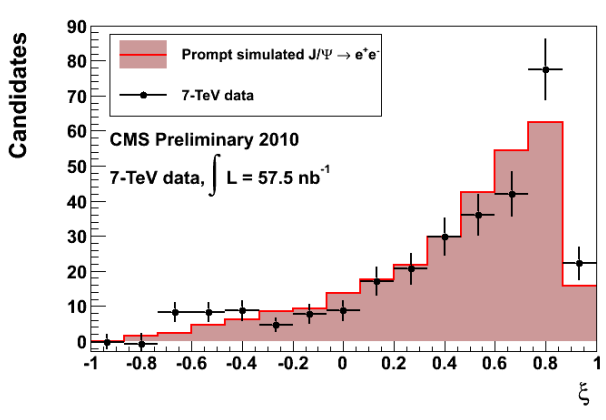

(b)

Figure 3: Invariant mass of the $\mathrm{J} / \Psi \rightarrow \mathrm{e}^{+} \mathrm{e}-$ candidates in the data (a). Distribution of the $\xi$ electron identification variable after background subtraction (b). The weighted $\xi$ distribution is compared with that obtained with a simulated sample.

The treatment of the muons is simpler than that of the electrons, as the reconstruction of a 
muon does not really depend on its isolation. Some care should, however, be taken in the algorithm so as to properly deal with the reconstructed track of a muon, and not treat it as a charged hadron. In addition, the muon selection criteria have been optimized for the best efficiency and the minimal fake rate in a large momentum range. The reliability of the muon reconstruction and of the particle-flow-based $E_{\mathrm{T}}^{\text {miss }}$ can be observed on Fig. 4 where the transverse mass distribution of events featuring a high- $p_{\mathrm{T}}$ muon exhibits a clear Jacobian peak from the $\mathrm{W} \rightarrow \mu \nu$ decays; no isolation is required on this plot. The data are well reproduced by the simulation both in the background and in the signal regions. Similar studies have been carried out in the electron channel, and give similar results.

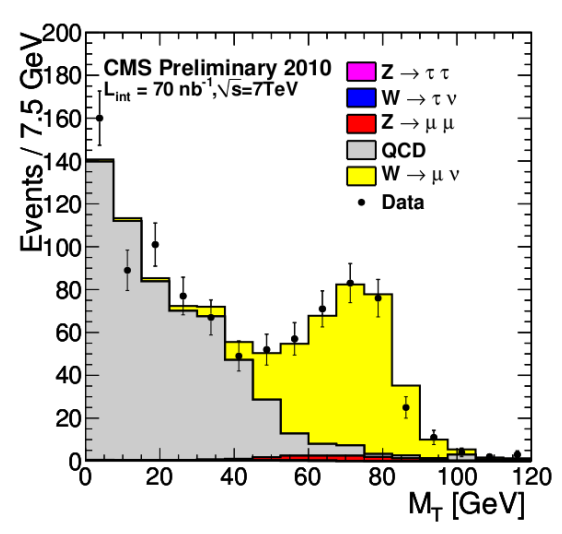

Figure 4: Distribution of the transverse mass reconstructed with the use of the particle-flow $E_{T}^{\text {miss }}$, without applying muon isolation.

\section{Conclusion}

Combining the various CMS sub-detectors, the particle-flow event reconstruction allows for a refined description of the event. The resolution of the jets thus reconstructed is improved by up to a factor three, and that of the $E_{\mathrm{T}}^{\text {miss }}$ is improved by a factor two compared to the calorimeter-based reconstruction. The commissioning of the algorithm is proceeding as expected and the energy scale of the jets, the $E_{\mathrm{T}}^{\text {miss }}$, the electrons, the photons, and muons is under control at the percent level.

\section{References}

[1] CMS Collaboration, E. Adolphi et al., The CMS experiment at the CERN LHC, JINST, 3 (2008) S08004.

[2] CMS Collaboration, Particle-Flow Event Reconstruction in CMS and Performance for Jets, Taus and $E_{\mathrm{T}}^{\text {miss }}, C M S$ PAS (2009) PFT-09-001.

[3] CMS Collaboration, Commissioning of the Particle-Flow reconstruction in Minimum-Bias and Jet Events from pp Collisions at 7 TeV, CMS PAS (2010) PFT-10-002.

[4] CMS Collaboration, Particle-Flow commissioning with muons and electrons from J/Psi, and W events at $7 \mathrm{TeV}$, CMS PAS (2010) PFT-10-003.

[5] M. Pivk and F. Le Diberder, A statistical tool to unfold data distributions, [hep-ph/ 0402083$]$ 